\title{
The Relativistic Space-Time Perspective
}

\section{David Grant Taylor}

Edmonton, Canada

Email: dgtaylor@telusplanet.net

How to cite this paper: Taylor, D.G. (2016) The Relativistic Space-Time Perspective. Journal of Modern Physics, 7, 2288-2298. http://dx.doi.org/10.4236/jmp.2016.716197

Received: August 20, 2016

Accepted: December 6, 2016

Published: December 9, 2016

Copyright $\odot 2016$ by author and Scientific Research Publishing Inc. This work is licensed under the Creative Commons Attribution International License (CC BY 4.0).

http://creativecommons.org/licenses/by/4.0/

\section{(c) (i) Open Access}

\begin{abstract}
Equations developed in this paper show the relationship between Real values measured from a Special Relativity Perspective with no Distortion [noSRPD] and the values measured from the same perspective when those values are distorted. Inverse equations using the values measured from distorted perspective [SRPD] are also reasoned. Equation logic proceeds from the original Real Time distortion equations from Einstein's On the Electrodynamics of Moving Bodies. An alternate Time equation using the distorted velocity measured from a Relativistic Perspective is presented. Since the time distortion range is unlimited, velocity from a Relativistic Perspective can have an infinite value. Eight additional formulas for velocity, mass, time, and linear deformations are reasoned. Relativistic Perspective equations have confirmed consistency with Classic Relativity equations to 2000 significant digits for 37 velocity values ranging from $1.0 \mathrm{E}-500 \mathrm{~m} / \mathrm{s}$ to $(\mathrm{c}-(1.0 \mathrm{E}-500)$ $\mathrm{m} / \mathrm{s}$.
\end{abstract}

\section{Keywords}

Perspective, Relativistic Distortion, Distorted Velocity, Parallel Equations, Confirmation Table

\section{Introduction}

Two equations developed in this paper show the velocity distortion from Special Relativity [SR] time effects from the distorted Perspective. The Classic Time equation in Einstein's On the Electrodynamics of Moving Bodies [1] is:

$$
\text { Time }^{\prime}=\text { Time } /\left(1-v^{2} / c^{2}\right)^{0.5}
$$

-where [Time] is the number of real seconds pass for any undistorted event, [Time'] is the number of real seconds that will pass under relativistic distortion for the same event, and $[\mathrm{V}]$ is the undistorted velocity. The existence of an ideal undistorted velocity 
is purely a theoretic proposal. Another phrasing would be [v] viewed from a Special Relativistic Perspective with no Distortion: [noSRPD]. So the variable name would be: $\left[v_{\text {noSRPD }}\right]$

$$
\text { Time }^{\prime}=\text { Time } /\left(1-v_{\text {noSRPD }}^{2} / c^{2}\right)^{0.5}
$$

Measuring velocity from a Special Relativistic Perspective with Distortion [SRPD] viewpoint can range from zero to infinity-limited noSRPD velocity with an unlimited time distortion. Or a value for a velocity $\left[v_{\text {SRPD }}\right]$ distorted to an infinite degree. The [Time] equation with that unlimited distortion would be:

$$
\text { Time }=\text { Time }^{\prime} /\left(1+v_{\text {SRPD }} / c^{2}\right)^{0.5}
$$

Consistency of the above to the Classic equations is confirmed to 2000 significant digits for 37 velocity values ranging from $1.0 \mathrm{E}-500 \mathrm{~m} / \mathrm{s}$ to $(c-1.0 \mathrm{E}-500) \mathrm{m} / \mathrm{s}$.

\section{Relativistically Distorted Velocities}

The SRPD||no SRPD relationship is derived from the [Time] equation. 'Real' labels for events are based on approximations. All objects in the universe are in motion, determining exact velocity from observation is impossible-so exact relativistic distortion is as well. Real is a theoretical state, not a confirmable one. But relativistic and non-relativistic relations are deducible. In

$$
\text { Time' }=\text { Time } /\left(1-v^{2} / c^{2}\right)^{0.5}
$$

[Time] is for episodes when viewers are not distorted. [Time'] for the same on a Relativistically distorted object. From an outside perspective, exchange of Bosons and their propagation velocity would slow. Current thinking is that from the moving object's perspective time would not slowdown-the objects/actions outside the distorted object would speed up.

An alternative would be relating seconds/time units passing on the distorted object to distance travelled. Real velocity is velocity with respect to an immobile point-a point with no Relativistic distortion. Currently, that would be an "impossible deal to close": an unattainable ideal. But it is conceivable and a methodology could be developed for achieving that ideal. Though [Time] could not be measured by a macro, multi-particle device. Relativistic distortions would change how that device functioned. Events would not proceed exactly as they do in a non-relativistic state. Matter particles would gain mass, and Boson particles would slow and weaken. Time passage would be fundamentally different from that of a non-Relativistic state. So define two new variables, recognizing the relativistic second as the inverse of a real second. As well, be more specific about the $[\mathrm{V}]$ variable, not as "Real" but as velocity undistorted by Relativistic effects. It presumes an ideal. Denying that premise would lead to discarding many motion, gravity and even Chemical reactions equations (relying on moles). Fewer relativistic seconds would pass once distortion began. The inverse would use SRPD values. 
Independent velocity would use both noSRPD time and noSRPD distance (metres).

The variable $v_{\mathrm{SRPD}}$ would be parallel to the value of the Real $|\nu|$ velocity perceived from a Relativistic viewpoint under no distortion. A $\left[\right.$ Time $_{\text {noSRPD }} \|$ Time $\left._{\text {SRPD }}\right]$ base is not an additional equation but the inverse of Einstein's Classic, Time units passing from the perspective of the moving object:

$$
\text { Time }_{\mathrm{SRPD}}=\mathrm{Time}_{\mathrm{noSRPD}} *\left(1-v_{\mathrm{noSRPD}}^{2} / c^{2}\right)^{0.5}
$$

Velocity is inversely to time. Divide both sides by 1 Real||noSRPD metre and invert $\left[1 \mathrm{~m} /\right.$ Time $\left._{\text {noSRPD }}\right]$ would mean the equation could determine relativistic vs. non-relativistic velocity instead of time distortion.

Define the $\left[\right.$ Time $\left._{\text {noSRPD }}\right]$ and the $\left[\right.$ Time $\left._{\text {SRPD }}\right]$ variable:

$$
\begin{gathered}
\text { Time }_{\text {noSRPD }}=1 \mathrm{~m}_{\text {noSRPD }} / v_{\text {noSRPD }} \\
\text { Time }_{\text {SRPD }}=1 \mathrm{~m}_{\text {noSRPD }} / v_{\text {SRPD }}
\end{gathered}
$$

Inversion would mean

$$
\begin{aligned}
v_{\text {noSRPD }} & =1 \mathrm{~m}_{\text {noSRPD }} / \text { Time }_{\text {noSRPD }} \\
v_{\text {SRPD }} & =1 \mathrm{~m}_{\text {noSRPD }} / \text { Time }_{\text {SRPD }}
\end{aligned}
$$

Divide both sides of the equation with 1 metre and invert that:

$$
\begin{aligned}
& \text { Time }_{\text {SRPD }} / 1 \mathrm{~m}_{\text {noSRPD }}=\left(\text { Time }_{\text {noSRPD }} / 1 \mathrm{~m}_{\text {noSRPD }}\right) *\left(1-v_{\text {noSRPD }}^{2} / c^{2}\right)^{0.5} \\
& 1 \mathrm{~m}_{\text {noSRPD }} / \text { Time }_{\text {SRPD }}=\left(1 \mathrm{~m}_{\text {noSRPD }} / \text { Time }_{\text {noSRPD }}\right) /\left(1-v_{\text {noSRPD }}^{2} / c^{2}\right)^{0.5}
\end{aligned}
$$

Using the above with the $\left[v_{\text {noSRPD }} \| v_{\text {SRPD }}\right]$ variables thus leads to Equation (1), the founding principle of Relativistic Perspective:

$$
v_{\mathrm{SRPD}}=v_{\mathrm{nOSRPD}} /\left(1-v_{\mathrm{nOSRPD}}^{2} / c^{2}\right)^{0.5}
$$

Everything in the universe has a velocity. Determining the speed of light [c] done from a viewpoint with an exactly zero velocity [2]. That is not possible anywhere in the Universe. So examine relativistic distortion from a theoretical perspective under no/ zero special relativistic distortion-noSRPD. Zero velocity may be indeterminate, but it is mathematically definable. A parallel would be the classic " $\mathrm{F}=$ ma" equation. All of the forces acting on a body can never be determined perfectly.

Classic Special Relativity presumes real values are values observed from a theoretic zero velocity. All observable objects in the universe are in motion. The maximum real velocity for matter objects or particles in our reality is agreed to be light velocity- $c$. That can also be argued to be a Planck velocity $\left[v_{p}\right]$ : dividing 1 Planck length $\left[l_{p}\right]$ or $\left[\left(\hbar G / c^{3}\right)^{0.5}\right]$ by a single Planck time $\left[t_{p}\right]$-or- $\left[\left(\hbar G / c^{5}\right)^{0.5}\right]$. Since both expressions hold the Gravitation Constant $[G]$ and the reduced Planck Constant $[\hbar]$, they cancel one another and the maximum Planck velocity can be reasoned to be:

$$
\begin{aligned}
& v_{p}=l_{p} / t_{p} \\
& v_{p}=\left(\hbar G / c^{3}\right)^{0.5} /\left(\hbar G / c^{5}\right)^{0.5} \\
& v_{p}^{2}=\left(\hbar G / c^{3}\right) /\left(\hbar G / c^{5}\right)
\end{aligned}
$$




$$
\begin{aligned}
& v_{p}^{2}=\left(1 / c^{3}\right) /\left(1 / c^{5}\right) \\
& v_{p}=c
\end{aligned}
$$

Since the Quantum level $[\hbar]$ constant is a determinant part of maximum velocity, so a reasonable postulate would be that the inverse is the minimum $\left[\right.$ Min $\left.\_v_{p}\right]-a$ Planck level velocity.

$$
\text { Min } \_v_{p}=1 / c=3.33553385 \mathrm{E}-9 \mathrm{~m} / \mathrm{s}
$$

An investigative Planck accurate timing device could measure its movement by a three-dimensional rotation, surveying all observable objects, measuring the red and blue shift on all. The observing device [O.D.] would exert a thrust to equalize those shifts. The O.D. would then survey another shift distortion, again thrusting in a vector that would equalize the distortion pattern. Repeat the above until any movement in any direction increased a blue shift from whatever was its value before that motion.

The experiment would be impossible methodologically, but is a valid ideal. Measurement of velocity from that point would give a Real velocity. Though the O.D.'s zero velocity point would only be for the moment it was marked. Movement of objects around the O.D. would change General relativistic distortions, twisting the shape of space-time. It would be a very theoretical but a valid ideal zero velocity. [ $\mathrm{F}=\mathrm{ma}$ ] is a parallel ideal: presuming exact formulaic measurements of only two bodies with no distortions. That condition does not exist anywhere in our reality. But those inaccuracies do not invalidate either equation.

\section{Non-Relativistic vs. Relativistic Velocities}

The non-Relativistic $\| v_{\text {noSRPD }}$ velocity can be made the dependent variable by inverting the velocity distortion equation. Start with squaring both sides:

$$
\begin{gathered}
v_{\mathrm{SRPD}}^{2}=v_{\mathrm{noSRPD}}^{2} /\left(1-v_{\mathrm{noSRPD}}^{2} / c^{2}\right) \\
v_{\mathrm{SRPD}}^{2} *\left(1-v_{\mathrm{nOSRPD}}^{2} / c^{2}\right)=\sim v_{\mathrm{SRPD}}^{2} *\left(1-v_{\mathrm{noSRPD}}^{2} / c^{2}\right) /\left(1-v_{\mathrm{noSRPD}}^{2} / c^{2}\right) \\
v_{\mathrm{SRPD}}^{2} *\left(1-v_{\mathrm{noSRPD}}^{2} / c^{2}\right)=v_{\mathrm{noSRPD}}^{2} \\
v_{\mathrm{SRPD}}^{2}-v_{\mathrm{SRPD}}^{2} * v_{\mathrm{noSRPD}}^{2} / c^{2}=v_{\mathrm{noSRPD}}^{2} \\
v_{\mathrm{SRPD}}^{2}-v_{\mathrm{SRPD}}^{2} * v_{\mathrm{noSRPD}}^{2} / c^{2}+\left(v_{\mathrm{SRPD}}^{2} * v_{\mathrm{noSRPD}}^{2} / c^{2}\right)=\sim v_{\mathrm{noSRPD}}^{2}+\left(v_{\mathrm{SRPD}}^{2} * v_{\mathrm{noSRPD}}^{2} / c^{2}\right) \\
v_{\mathrm{SRPD}}^{2}-v_{\mathrm{SRPD}}^{2} * v_{\mathrm{noSRPD}}^{2} / c^{2}=v_{\mathrm{noSRPD}}^{2} \\
v_{\mathrm{SRPD}}^{2}=v_{\mathrm{noSRPD}}^{2}+v_{\mathrm{SRPD}}^{2} * v_{\mathrm{noSRPD}}^{2} / c^{2} \\
v_{\mathrm{SRPD}}^{2}=v_{\mathrm{noSRPD}}^{2} *\left(1+v_{\mathrm{SRPD}}^{2} / c^{2}\right) \\
v_{\mathrm{noSRPD}}^{2}=v_{\mathrm{SRPD}}^{2} /\left(1+v_{\mathrm{SRPD}}^{2} / c^{2}\right) \\
v_{\mathrm{noSRPD}}=v_{\mathrm{SRPD}} /\left(1+v_{\mathrm{SRPD}}^{2} / c^{2}\right)^{0.5}
\end{gathered}
$$

Again, Equations ((1) and (2)) have been table confirmed for velocities ranging from $1.00 \mathrm{E}-500 \mathrm{~m} / \mathrm{s}$ to $[\mathrm{c}-1.00 \mathrm{E}-500] \mathrm{m} / \mathrm{s}$ to 2000 decimal places. The velocity equations 
have another form with division of both sides of Equations $\left((1)\right.$ and (2)) with $v_{\text {noSRPD }}$ :

$$
\begin{aligned}
& v_{\mathrm{SRPD}} / v_{\text {noSRPD }}=\left(v_{\text {noSRPD }} /\left(1-v_{\text {noSRPD }}^{2} / c^{2}\right)^{0.5}\right) / v_{\text {noSRPD }} \\
& v_{\text {SRPD }} / v_{\text {noSRPD }}=1 /\left(1-v_{\text {noSRPD }}^{2} / c^{2}\right)^{0.5} \\
& v_{\text {noSRPD }} / v_{\text {SRPD }}=\left(1-v_{\text {noSRPD }}^{2} / c^{2}\right)^{0.5}
\end{aligned}
$$

And by dividing both sides of Equation (2) with $v_{\mathrm{SRPD}}$ :

$$
\begin{aligned}
& v_{\text {noSRPD }} / v_{\text {SRPD }}=\left(v_{\mathrm{SRPD}} /\left(1+v_{\mathrm{SRPD}}^{2} / c^{2}\right)^{0.5}\right) / v_{\mathrm{SRPD}} \\
& v_{\mathrm{nOSRPD}} / v_{\mathrm{SRPD}}=1 /\left(1+v_{\mathrm{SRPD}}^{2} / c^{2}\right)^{0.5} \\
& v_{\mathrm{SRPD}} / v_{\mathrm{noSRPD}}=\left(1+v_{\mathrm{SRPD}}^{2} / c^{2}\right)^{0.5}
\end{aligned}
$$

These proportions mean the $\left(1-v_{\text {noSRPD }}^{2} / c^{2}\right)^{0.5} \|\left(1+v_{\mathrm{SRPD}}^{2} / c^{2}\right)^{0.5}$ expressions can be interchanged.

Gravitational and Special Relativistic distortion are part of all reality. The Real $[v]$ value is always approximate. Terms should not be Relativistic and Real, but Relativistic and non-Relativistic. Any observed velocity is valid as a Relativistic velocity. The sole issue is the precision of the value. Lower velocities use noSRPD values, higher velocities use SRPD for conversion to non-Relativistic values. Though neither measurement will ever be exact.

The above thought experiment presumes undistorted measurements, presumed ideals. Zero velocity will always be an unreachable ideal. Sufficient data may be a theoretically reachable goal, but gathering such data would require multiple observation points in distinct and separate (on an intra-galactic scale) locations. There is also the difficulty of moving a sophisticated observation device at a relativistic velocity for observations of distortions from the relativistic viewpoint. This velocity would distort different variables in different ways. The most obvious examples are the mass of the matter and the velocity of boson particles. The matter would increase in mass, but bosons would decrease in velocity and mass. The relationship between the two quantities would become dysfunctional. The elements would dissemble to their component protons, neutrons and electrons, because the gluons could be weakened to an infinitesimal degree. The repulsive force of the positive charge would weaken to the same degree, but mass of the nucleons together would increase accordingly. So any passenger aboard a vessel moving at a relativistic velocity would both gain weight and lose muscular force.

\section{Additional Relativistic Equations}

The Relativistic Perspective velocity formulae can be used to deduce the same for time, length, and mass. The Classic time distortion equation, in Relativistic Perspective form can be written:

$$
\text { Time' }=\text { Time } /\left(1-v_{\text {noSRPD }}^{2} / c^{2}\right)^{0.5}
$$




$$
\begin{gathered}
\text { Time }^{\prime}=\text { Time } /\left(v_{\text {noSRPD }} / v_{\text {SRPD }}\right) \\
\text { Time }=\text { Time }^{\prime} /\left(v_{\text {SRPD }} / v_{\text {noSRPD }}\right) \\
\text { Time }=\text { Time }^{\prime} /\left(1+v_{\text {SRPD }}^{2} / c^{2}\right)^{0.5}
\end{gathered}
$$

And the inverse Relativistic Perspective equations:

$$
\begin{gathered}
\text { Time }_{\text {SRPD }}=\text { Time }_{\text {noSRPD }} *\left(1-v_{\text {noSRPD }}^{2} / c^{2}\right)^{0.5} \\
\text { Time }_{\text {noSRPD }}=\text { Time }_{\text {SRPD }} *\left(1+v_{\text {SRPD }}^{2} / c^{2}\right)^{0.5}
\end{gathered}
$$

The other equations have the same logic. $\left[\mathrm{Mass}_{\text {nosRPD }}\right]$ would be the mass of any object when there was no distortion, and $\left[\mathrm{MaSS}_{\mathrm{SRPD}}\right]$ what it was under distortion.

$$
\begin{aligned}
& \operatorname{MaSS}_{\mathrm{SRPD}}=\operatorname{MasS}_{\mathrm{noSRPD}} /\left(1-v_{\mathrm{noSRPD}}^{2} / c^{2}\right)^{0.5} \\
& \operatorname{MaSS}_{\mathrm{SRPD}}=\operatorname{MaSS}_{\mathrm{noSRPD}} /\left(v_{\text {noSRPD }} / v_{\mathrm{SRPD}}\right) \\
& \text { MaSS }_{\text {noSRPD }}=\operatorname{MaSS}_{\mathrm{SRPD}} /\left(v_{\mathrm{SRPD}} / v_{\mathrm{noSRPD}}\right) \\
& \operatorname{Mass}_{\text {noSRPD }}=\operatorname{MaSS}_{\text {SRPD }} /\left(1+v_{\text {SRPD }}^{2} / c^{2}\right)^{0.5}
\end{aligned}
$$

The length equation that was the very first movement in the development of the Relativity equations:

$$
L=L_{0} /\left(1-v^{2} / c^{2}\right)^{0.5}
$$

where $\left[L_{0}\right]$ would be the length (on the same vector as the velocity) when the object was at rest and $[L]$ the length once the object started to move. Using more specific SRPD variable definitions for Length equations: an SRPD viewpoint with no distortion $\left[\right.$ Length $\left._{\text {noSRPD }}\right]$ and $\left[\right.$ Length $\left._{\mathrm{SRPD}}\right]$ would be the value when there was.

$$
\begin{aligned}
& \text { Length }_{\mathrm{SRPD}}=\text { Length }_{\mathrm{noSRPD}} *\left(1-v_{\mathrm{noSRPD}}^{2} / c^{2}\right)^{0.5} \\
& \text { Length }_{\mathrm{SRPD}}=\text { Length }_{\mathrm{noSRPD}} *\left(v_{\mathrm{noSRPD}} / v_{\mathrm{SRPD}}\right) \\
& \text { Length }_{\mathrm{noSRPD}}=\text { Length }_{\mathrm{SRPD}} *\left(v_{\mathrm{SRPD}} / v_{\mathrm{noSRPD}}\right) \\
& \text { Length }_{\mathrm{noSRPD}}=\text { Length }_{\mathrm{SRPD}} *\left(1+v_{\mathrm{SRPD}}^{2} / c^{2}\right)^{0.5}
\end{aligned}
$$

Velocity can appear to reach or exceed the speed of light from the moving body's perspective because of relativistic distortions. Relativistic Perspective equations determine the relativistic distortions from moving observation points.

The Einsteinian equations are more appropriate for low speeds. Motion is relative for any observation point; determining an exact "velocity" is impossible. Alternately, if all observed objects exhibit a large blue shift (including a point where that shift was the greatest) that would indicate a need for Relativistic Perspective Equations. Relativistic perspective equations determine the appropriate relativistic values (for the velocity, time, mass, and length) from the corresponding non-relativistic values. 


\subsection{Other Consequences of Relativistic Distortion}

The above equations suggest additional relativistic effects not currently recognized by science.

Relativistic effects slow the transmission of all Bosons: Photons (light), Gravitons (gravity), Gluons (strong nuclear force) and the W/Z Bosons (weak nuclear force). Bosons emitted by any moving object will be slowed down by those distortions, reducing the frequency-reducing those bosons' mass as well.

Relativistic effects also increase the mass of matter particles; the mass transferred from the bosons. If the acceleration is done from outside the moving object, because that energy is coming from outside the object it will increase the mass of the Bosons on a Relativistic scale. But in a macro level object, particles/atoms/molecules would increase in mass, and the velocity of Bosons generated by those would reduce by exactly the same proportion. But because they were generated by the mass increased matter particles, the overall energy of those Bosons would increase and so the structural energy for any quantum, atomic or molecular particulates would remain the same. But it would be acting with matter increased in mass. The kinetic energy of those particulate objects would increase by the same proportion. A good parallel for that would be an increase in temperature-the greater the speed of the object, the greater the kinetic energy of its particulates. The velocity of all the structural Bosons would slow, but the matter they maintained they would also increase in temperature.

Specifically, the principle Hydrogen isotope $-{ }^{1} \mathrm{H}$ - has an atomic mass of $1.0079-$ fundamentally different nucleic structure from the principle Iron isotope $-{ }^{56} \mathrm{Fe}-$ one that has an atomic mass of 55.845 . A velocity of $2.59627884 \mathrm{E}^{8} \mathrm{~m} / \mathrm{s}$ means an approximate distortion factor of 2.0. That velocity would mean the mass of individual nucleons would be doubled while the mass of Bosons is halved. Would the interactions of ${ }^{1} \mathrm{H}$ with an atomic mass of 2.0158 (or ${ }^{56} \mathrm{Fe}$ with an atomic mass of 111.869 ) remain exactly the same, only slower? Doubling the nucleon mass and halving Gluon velocities to $149,896,229 \mathrm{~m} / \mathrm{s}$ ?

Quantum-level interactions would be dealing with heavier particles with slower, less energetic Boson forces. Time would not slow in progression; the interactions that maintain the Quantum structure of any macro-level device would slow. There would be a fundamental difference in the way Bosons and matter particles interacted. It would change the fundamentals of the forces that maintain the reality we observe-changing how that reality progresses.

In the great "Time" debate about Relativity this paper aggressively does not take either side: in some respects it would be as though time were slowing down. But quantum values of everything would change.

Alterations occurring at a low distortion level would change fundamental quantum interactions but only to a marginal degree. An observable relativistic scale recession velocity could alternately indicate relativistic scale distance and boson decay through matter interactions, not a universal expansion: EM frequency decay over cosmological distances through the passage of extremely disperse matter clouds that are reasoned to 
exist throughout our reality. The frequency-decay supposition does not conflict with any conservation principles currently accepted in science. Through the acceleration of the objects, a very fundamental fact of our observations has already been quantified: the Hubble Constant. That would mean an increase in the energy level of the Universe, with no limit or source for that increase. While the universe's expansion principle contradicts the one of the most fundamental principles in modern Science (matter/energy conservation) frequency decay does not. The EM decay through disperse matter would not be an absolute reduction of the energy in any EM signal; it would be its partial transformation into kinetic energy for that matter.

\subsection{An Uncertain Speculation}

In our reality, Science reasons that the maximum possible velocity from an undistorted Perspective is $c$-light velocity. The reader may feel compelled to debate other assertions in this paper. But surely the above can be taken as incontestable because so many physics principals rely on that maximum. Another argument for that limit can be made with the uncertainty principle.

If the inverse of the speed of light is a Quantum minimum, then it is reasonable to propose that it is absolute slowest that energy Bosons could move before they displayed matter like uncertainty. The principle of matter/energy conservation would mean that the low energy Bosons would increase to the maximum number there could be for any defined amount of energy. All of those would be Bosons moving at the slowest possible speed. So the individual said Bosons would be approaching zero rest mass.

General Relativity argues the same Boson slowdown, so the above will be of use in reasoning parallel events in gravitational distortion. By current theory, Special Relativistic effect could produce the above state. If the maximum Real, non-Relativistic velocity could produce a distortion that would lower Boson velocity from $c$ to $[1 / c]-$ a distortion of $\left[1 / c^{2}\right]$. Distortion shifting Boson velocity to its antithesis-the inverse of light speed. Any greater shift would mean that Boson velocity would become imaginary -the antithesis of Time||Velocity distortion for matter. The slowest that any Boson could move. The formula determining that distortion would be

$$
\begin{aligned}
& 1 / c=c *\left(1-v_{\mathrm{noSRPD}}^{2} / c^{2}\right)^{0.5} \\
& 1 / c^{2}=c^{2} *\left(1-v_{\mathrm{noSRPD}}^{2} / c^{2}\right) \\
& 1 / c^{4}=1-v_{\mathrm{nOSRPD}}^{2} / c^{2} \\
& 1 / c^{4}-1=-v_{\mathrm{noSRPD}}^{2} / c^{2} \\
& v_{\mathrm{noSRPD}}^{2}=c^{2} *\left(1-1 / c^{4}\right) \\
& v_{\text {noSRPD }}=c^{*}\left(1-1 / c^{4}\right)^{0.5}
\end{aligned}
$$

The $v_{\text {noSRPD }}$ needed to produce that distortion (to 50 decimal places) would be:

$$
v_{\text {noSRPD }}=299,792,458 *\left(1-1 / 299,792,458^{4}\right)^{0.5}
$$




$$
v_{\text {noSRPD }}=2.99792457999999999999999999999999981442994539015080 \mathrm{E} 8
$$

So at the above velocity the distortion could be proposed as a maximum [Max_Dist $\left.{ }_{\hbar}\right]$, and bosons would move at approximately:

$$
\operatorname{Max} \_ \text {Dist }_{\hbar}=\left(1-v_{\text {noSRPD }}^{2} / c^{2}\right)^{0.5}
$$

$$
\begin{aligned}
& \text { Max_Dist }_{\hbar}=\left(1-2.9979245799 \sim 9981442994539015080 \mathrm{E} 8^{2} / c^{2}\right)^{0.5} \\
& \text { Max_Dist }_{\hbar}=1.11265005605361843217408996484800999160753243447496 \mathrm{E}-17
\end{aligned}
$$

The velocity of individual energy Bosons would decrease to that degree. The mass of fundamental matter particles (Electrons, Protons, Neutrons) would increase by the inverse by the square of $\mathrm{c}$ would be less irrational, because it would not involve divisionan energy to matter distortion [e_m $\left.\mathrm{m}_{\text {Dist }}\right]$ factor of $c^{2}$.

$$
\text { e_m } \mathrm{m}_{\text {Dist }}=c^{2}=\sim 8.98755178736817640000000000000000000000000000000000 \mathrm{E} 16 \text {. }
$$

Matter would absorb the Energy of Bosons in the distorted environment. Because of the inevitable transformation to matter, it is reasonable to say that the Bosons would reach their greatest similarity at the above velocity. A question then arises: would a boson energy decrease factor of 1.112650056 243447496E-17 and a matter particulate increase factor of $8.987551787368176400 \sim 00 \mathrm{E} 16$ - result in a simple slow down in time? The combined Max Planck Slowdown||Decrease Distortion of Bosons divided by the mass increase distortion factor of the matter-Comb $b_{\text {Dist }}$ :

$$
\begin{gathered}
\text { Comb }_{\text {Dist }}=\text { Max_Dist }_{\hbar} /{\mathrm{e} \_\mathrm{m}_{\text {Dist }}} \\
\mathrm{Comb}_{\text {Dist }}=(1.1126500560 \sim 43447496 \mathrm{E}-17) /(8.987551787368176400 \sim 00 E 16) \\
\mathrm{Comb}_{\text {Dist }}=1.2379901472361202391251417385431601832158 \sim 3913048221 \mathrm{E}-34
\end{gathered}
$$

Would Quantum scale events be fundamentally different in a vessel moving at the above velocity than ones that take place in a body with a Relativistic ideal zero motion? The cumulative effect being that Bosons could not be more matter like in their aspect at that point, and could transform at some point in the Boson slowdown so that their energy Quanta aspects were overpowered by their matter Quanta aspects. The above could be reasoned to be a possible link between Relativity and Quantum Physics.

The velocity of individual energy Bosons would decrease to that degree. The mass of fundamental matter particles (Electrons, Protons, Neutrons) would increase by the inverse.

Matter would absorb the Energy of Bosons in the distorted environment. Because of the inevitable transformation to matter, it is reasonable to say that the Bosons would reach their greatest similarity at the above velocity. A question then arises: would matter particles increasing by factor of 8.98753269 - 999999992E16 manipulated by Bosons with an energy decrease factor of 1.11265238 - 202746730E-17 be a simple slow down in time? The matter/energy proportion would change by a factor of $1.237990147227 \mathrm{E}-34$. Would Quantum scale events be fundamentally different in a vessel moving at the above velocity than ones that take place in a body with a Relativistic ideal zero motion? The cumu- 
lative effect being that Bosons could not be more matter like in their aspect at that point, and could transform at some point in the Boson slowdown so that their energy Quanta aspects were overpowered by their matter Quanta aspects. The above could be reasoned to be a possible link between Relativity and Quantum Physics.

Also consider the relatively infinitesimal differences in the actual atom mass of varying flavours elements Atoms/Isotopes and the even more infinitesimal differences in the mass of nucleic particles within those atoms mean very significant differences in the properties of those elements. At the simple speed of $295,000,000 \mathrm{~m} / \mathrm{s}$, the degree of distortion $\left[\right.$ Degree $\left._{\text {Dist }}\right]$ would be

$$
\begin{aligned}
& \text { Degree }_{\text {Dist }}=1 /\left(1-2.9500 \mathrm{E} 08 / c^{2}\right)^{0.5} \\
& \text { Degree }_{\text {Dist }}=5.615
\end{aligned}
$$

The paired forces that maintain the structure of the nucleus of any Element, the outward pressure of the matching Proton charges and the inward pressure of the Strong Nuclear Force Gluons would both reduce by approximately the above factor of 5.615. The same distortion factor would apply to mass of the particles generating Bosons. The most stable element in our Science, the Iron-56 isotope has a rest mass of approximately 55.846. At the above velocity it would have a mass of more 313.576 Atomic mass units. An element with a nuclear mass of more than 313 atomic units while the Photon and the Gluon forces that maintained its structure were the same as the isotope at rest would not have the same chemical or nuclear properties.

There can be no assertions made as to the rate at which energy particles are transformed into matter-that issue would not be resolved without further experimental investigation and theoretical postulates. But the above reasons additional aspects of the energy to matter process on a Quantum level. It would also mean that different Bosons would become more and more alike as they transformed into matter.

\section{Summary}

This paper has formulated additional relativistic equations that do not contradict Special Relativity. They are the same equations from a relativistic viewpoint. The equations presented examine special relativistic distortions from the perspective of the distorted object, and they determine the non-relativistic velocity from the observed velocity in the moving object. The values of the non-relativistic velocity and the apparent relativistic velocity it engenders share exactly the same validity. The equations relating these two perspectives are documented in this paper, the most crucial being:

$$
\text { Time }=\text { Time }^{\prime} /\left(1+v_{\mathrm{SRPD}}^{2} / c^{2}\right)^{0.5} \text {. }
$$

The distortion in Time, Time', Velocity (and in all other Relativistic quantities) can be distorted to a theoretically infinite degree. An equation for the antithesis of the Real Time distortion must be logically structured to allow for that. Those Relativistic effects may distort Quantum values for Boson||Energy particles to a degree that they became indistinguishable. 


\section{References}

[1] Einstein, A. and der Physik, A. (1905) Annals of Physics, 322, 891-921.

http://hermes.ffn.ub.es/luisnavarro/nuevo_maletin/Einstein_1905_relativity.pdf

[2] Planck, M. (1901) Annalen der Physik, 309, 553-563.

https:/doi.org/10.1002/andp.19013090310

\section{Submit or recommend next manuscript to SCIRP and we will provide best service} for you:

Accepting pre-submission inquiries through Email, Facebook, LinkedIn, Twitter, etc. A wide selection of journals (inclusive of 9 subjects, more than 200 journals)

Providing 24-hour high-quality service

User-friendly online submission system

Fair and swift peer-review system

Efficient typesetting and proofreading procedure

Display of the result of downloads and visits, as well as the number of cited articles

Maximum dissemination of your research work

Submit your manuscript at: http://papersubmission.scirp.org/

Or contact jmp@scirp.org 